\title{
Two cases of neonatal adrenal hemorrhage presenting with persistent jaundice
}

\author{
Due casi di emorragia surrenalica neonatale manifestatasi con ittero persistente
}

E. Ruffini, ${ }^{1}$ L. De Petris,${ }^{1}$ G. Zorzi, ${ }^{1}$ P. Paoletti, ${ }^{2}$ G. Mambelli, ${ }^{2}$ A. Carlucci ${ }^{1}$

Key words: neonatal adrenal hemorrhage, persistent jaundice, cystic neuroblastoma, ultrasonography

\begin{abstract}
The adrenal hemorrhage is a relatively rare event in newborns but must be considered in the presence of a persistent unexplained jaundice, especially in presence of predisposing factors. Serial ultrasonography is the modality of choice for initial diagnosis and follow-up of neonatal adrenal hemorrhage.

We report two cases of neonatal adrenal hemorrhage presenting with persistent jaundice. The causes of the neonatal adrenal hemorrhages were a difficult vaginal delivery in macrosomic infant and a neonatal infection.
\end{abstract}

\section{Riassunto}

L'emorragia surrenalica è un evento relativamente raro nei neonati ma deve essere considerata in presenza di ittero persistente inspiegabile, specialmente in presenza di fattori predisponenti. L'esame ecografico seriato è la modalità di prima scelta per la diagnosi iniziale e il follow-up dei neonati con emorragia surrenalica. Riportiamo due casi di emorragia surrenalica neonatale manifestatesi con ittero persistente. Le cause correlate ai due casi di emorragia surrenalica presentati erano un parto vaginale difficile di un neonato macrosoma e un' infezione neonatale.

\footnotetext{
1 Pediatrics and Neonatology Division, Maternal and Infant Department, Mazzoni Hospital, Ascoli Piceno, Italy

2 Radiology Division, Diagnostic Imaging Department, Mazzoni Hospital, Ascoli Piceno, Italy
}

Indirizzo per la corrispondenza (Corresponding author):

E. Ruffini

Pediatrics and Neonatology Division, Maternal and Infant Department, Mazzoni Hospital Via degli Iris, 1 - 63100 Ascoli Piceno - Italy

E-mail: eruffini@libero.it

\section{Introduction}

Adrenal hemorrhage $(\mathrm{AH})$ is unusual in infants and occurs most often after a traumatic delivery or a complicated neonatal course. The most common clinical symptoms are abdominal mass, jaundice and mild anemia. Persistent neonatal jaundice is rarely the primary presentation. ${ }^{1}$ The finding of an adrenal mass may lead to problems of differential diagnosis with other serious illnesses such as cystic neuroblastoma. ${ }^{2}$ We report two cases of neonatal $\mathrm{AH}$ confirmed by abdominal ultrasonography (US) and magnetic resonance imaging (MRI) presenting with persistent jaundice. The causes of neonatal $\mathrm{AH}$ were a difficult vaginal delivery in a macrosomic infant and a neonatal infection.

\section{Case Report}

Case 1

A two-day-old male infant was transferred to our hospital with a fever, elevated CRP and thrombocytopenia. In another medical center, the child was born by normal spontaneous vaginal delivery at $39+1$ weeks of gestation after an uneventful pregnancy. Birth weight was $4100 \mathrm{~g}$ and APGAR scores were 8 and 10 at 1 and 5 minutes, respectively.

Systemic examination was normal except for icterus and irritability. Laboratory results confirmed the increase of CRP $(10 \mathrm{mg} / \mathrm{dl})$ and thrombocytopenia $\left(91 \times 10^{6} / \mathrm{l}\right)$ with normal coagulation parameters and blood chemistry.

Therapy was started with Ampicillin and Gentamycin. At three days of life, high values of serum bilirubin $(15,3 \mathrm{mg} / \mathrm{dl})$ with direct fraction of $0,9 \mathrm{mg} / \mathrm{dl}$ phototherapy was started. Within a few days the CRP and platelet levels normalized, while blood and CSF cultures were negative. Antibiotic therapy was discontinued after 10 days. At 15 days of life, elevated total serum bilirubin $(18,4 \mathrm{mg} / \mathrm{dl})$ with direct bilirubin $0.6 \mathrm{mg} / \mathrm{dl}$ persisted. The infant was fed formula milk. Both baby and mother had A+ blood group and Coombs test was negative. Laboratory studies revealed hemo- 
globin of $13,5 \mathrm{~g} / \mathrm{dl}$, reticulocyte count of $1,5 \%$ and liver function tests were normal. Urine analysis and culture were normal and the urine tested negative for reducing substances. Serum glucose-6phosphate dehydrogenase and thyroid concentrations were all normal. The Cranial ultrasonography (US) was normal but abdominal US revealed a heterogeneous soft-tissue mass lesion consistent with an adrenal hematoma $35 \mathrm{~mm}$ right and $29 \mathrm{~mm}$ to the left. Magnetic resonance imaging (MRI) of the abdomen confirmed the picture without enhancement and/or calcifications. 24-hour urinary vanillylmandelic acid/catecholamine levels were normal. Serial ultrasound examinations showed significant reduction of AH. At 20 weeks of life, abdominal US showed slight increase in volume of the adrenal gland, in particular on the right, but no structural alterations.

\section{Case 2}

A nineteen-day-old female infant, fed formula milk, was admitted to our hospital for jaundice. The child was born at $41+2$ weeks gestation to a 36-year old woman after an uneventful pregnancy. A difficult vaginal delivery, carried out without instrumental assistance, led to a fracture of the infant's right clavicle. Birth weight was 5150 $\mathrm{g}$ and APGAR scores were 9 and 10, at 1 and 5 minutes, respectively. She was discharged on fourth day with total serum bilirubin $12,6 \mathrm{mg} / \mathrm{dl}$.

The infant's weight was $5210 \mathrm{~g}$ and systemic examination was normal except for icterus and a hard lump on the right clavicle. Laboratory studies revealed: total serum bilirubin of $18,6 \mathrm{mg} / \mathrm{dl}$; direct bilirubin of $0.5 \mathrm{mg} / \mathrm{dl}$.; hemoglobin: $14,8 \mathrm{~g} / \mathrm{dl}$; reticulocyte count of $2 \%$; and liver function tests were normal. Both baby and mother had $0+$ blood group and infant was Coombs negative. Urine analysis and culture were normal and the urine tested negative for reducing substances. Serum glucose-6-phosphate dehydrogenase and thyroid concentrations were all normal.

Cranial US was normal but abdominal US revealed an oval mass of $42 \mathrm{~mm}$, a prevalently solid structure of the right adrenal gland. This picture was confirmed by abdominal MRI. There was no enhancement in the post-contrast or calcification in the mass (figure 1). Coagulogram and 24-hour urinary vanillylmandelic acid/catecholamine levels were normal. Serial ultrasound examinations showed significant reduction of AH (figure 2). At 16 weeks old, the abdominal US was normal.

\section{Discussion}

Persistent jaundice in the neonate is defined as jaundice that lasts longer than 14 days; it can occur in up to $15 \%$ of all newborns. The vast majority of these neonates have unconjugated hyperbilirubinemia and the most known causes include breast milk jaundice, haemolytic diseases, galactosemia, hypothyroidism, urinary tract infection and blood extravasation. ${ }^{3}$

The main guidelines for the management of an infant with persistent jaundice are reported among the most common forms of blood extravasation, cephalohematoma and significant bruising. There is no data pertaining to other sites where internal hematomas may occur such as spleen, liver, internal cranium and adrenal glands. ${ }^{4}$ Although $\mathrm{AH}$ is an unusual event in newborns, it may present with persistent jaundice. $^{1}$

AH usually occurs at birth or during the immediate postnatal period and the incidence ranges from approximately 1.7 to 2.1 per 1000 births. It affects the right side more than the left side and is bilateral in 10 to $15 \%$ of the cases. In the newborn, the adrenal gland is very large compared to weight and unique vascular supply; for this reason it is more vulnerable to vascular damage. Any condition leading to hypoxia may lead to shunting of blood flow to vital organs. The increased pressure associated with congestion and the damaged endothelial cells associated with hypoxia may cause $\mathrm{AH} .{ }^{1}$ Birth trauma, prolonged labor, intrauterine infection, perinatal asphyxia or hypoxia, large birth weight, septicemia, hemorrhagic disorder and hypothrombinemia are the most common predisposing factors of $\mathrm{AH} .{ }^{1}$

Clinical manifestations of adrenal hemorrhage can vary depending on the degree and rate of hemorrhage, as well as the amount of adrenal cortex compromised by the hemorrhage. Although an isolated focal unilateral AH may present subclinically, massive bilateral AH may present in shock. Large unilateral or bilateral extensive bleeds may also present with palpable flank mass, mild anemia, jaundice or scrotal hematoma. ${ }^{5,6}$ Addisonian crisis is rare in $\mathrm{AH}$ because hemorrhage is predominantly subcapsular and adrenal insufficiency does not occur until at least $90 \%$ of adrenal tissue is destroyed.?

Neonatal jaundice is common and occurs due to hemolysis from enclosed hemorrhage. Persistent neonatal jaundice is rarely the primary presentation. ${ }^{8,9}$

The finding of an adrenal mass may lead to serious problems of differential diagnosis as other serious pathological entities such as cystic neuroblastoma must be considered. ${ }^{2,10}$

Postnatal diagnosis of cystic neuroblastoma may be obtained by indirect signs such as collection of 24-h urine specimen to measure vanillylmandelic acid, and by radiological examinations such as US or MRI. ${ }^{2,11}$ Although the imaging results of two groups of cystic lesions were similar, the failure of prenatal detection, the absence of calcification on initial images, and the size reduction are all factors that suggest $\mathrm{AH} .{ }^{12,13}$ For these reasons, serial US is the best modality for monitoring $\mathrm{AH}$ to prevent unnecessary surgery. ${ }^{14}$

In our patient VMA levels were normal; there was no calcification, and follow-up US revealed decrease in size of the cyst.

\section{Conclusion}

The possibility of AH as a cause of persistent jaundice should not be ignored, especially in children with predisposing factors. In addition, the importance of a correct differential diagnosis with cystic neuroblastoma through a follow-up US is emphasized. 


\section{References}

1 Mangurten HH. Birth injuries. In: Martin RJ, Fanaroff AA, Walsh MC (eds). Fanaroff and Martin's Neonatal Perinatal Medicine-Diseases of the Fetus and Newborn (8th ed). Philadelphia: Mosby Elsevier; 2006: 529-59.

2 Eo H, Kim JH, MD1, Jang KM et al. Comparison of Clinico-radiological Features between Congenital Cystic Neuroblastoma and Neonatal Adrenal Hemorrhagic Pseudocyst. Korean J Radiol 2011;12(1):52-58

3 Gilmour SM. Prolonged neonatal jaundice: When to worry and what to do. Paediatr Child Health 2004; 9: 700-4.

4 Porter ML, Dennis BL. Hyperbilirubinemia in the term newborn. Am Fam Physician 2002; 65: 599-607.

5 Avolio L, Fusillo M, Ferrari G, Chiara A, Bragheri R. Neonatal adrenal hemorrhage manifesting as acute scrotum: timely diagnosis prevents unnecessary surgery. Urology 2002; 59: 601.

6 Miele V, Galluzzo M, Patti G, Mazzoni G, Calisti A, Valenti M. Scrotal hematoma due to neonatal adrenal hemorrhage: the value of ultrasonography in avoiding unnecessary surgery. Pediatr Radiol 1997;27:672-74.
7 Simon DR, Palese MA. Clinical update on the management of adrenal hemorrhage. Curr Urol Rep.2009;10:78-83.

8 American Academy of Pediatrics Subcommittee on Hyperbilirubinemia. Management of hyperbilirubinemia in the newborn infant 35 or more weeks of gestation. Pediatrics 2004;114:297-316.

9 Maisels MJ, Kring E. Rebound in serum bilirubin level following intensive phototherapy. Arch Pediatr Adolesc Med 2002;156:669-72.

10 Eklof O, Mortensson W, Sandstedt B. Suprarenal haematoma versus neuroblastoma complicated by haemorrhage: a diagnostic dilemma in the newborn. Acta Radiol Diagn 1986;27:3-10.

11 Kawashima A, Sandler CM, Ernst RD et al. Imaging of nontraumatic hemorrhage of the adrenal gland. Radiographics 1999;19: 949-63.

12 Koplewitz BZ, Daneman A, Cutz E Hellmann J. Neonatal adrenal congestion: a sonographic-pathologic correlation. Pediatr Radiol 1998;28:958-62.

13 Velaphi SC, Perlman JM. Neonatal adrenal hemorrhage: clinical and abdominal sonographic findings. Clin Pediatr 2001;40:545-48.

14 Bergami G, Malena S, Di Mario M et al. Sonographic follow-up of neonatal adrenal hemorrhage. Fourteen case reports. Radiol Med 1990; 79:474-78. 